\title{
MARCA GRÁFICA CHAKIÑAN: PROPUESTA DESDE EL SISTEMA CO-CREATIVO O DISEÑO COLABORATIVO
}

\author{
CHAKIÑAN GRAPHIC BRAND: PROPOSAL FROM THE \\ CO-CREATIVE SYSTEM OR COLLABORATIVE DESIGN
}

\section{RESUMEN}

La naciente revista científica de ciencias sociales y humanidades, Chakiñan, requería una identificador visual que represente su identidad, adquiera notoriedad entre la comunidad académica y logre posicionarse en el contexto de las revistas indexadas. Para cumplir la citada tarea se propuso el sistema de diseño colaborativo, con la participación de ocho estudiantes que cursaron la asignatura de Diseño Andino en el periodo octubre 2016 - marzo 2017. En el desarrollo se empleó la metodología proyectual de Bruce Archer que contempla cuatro fases: analítica, creativa, formalización de la idea y fase ejecutiva. Entre las condicionantes se contempló la necesidad que, el identificador visual refleje la cosmovisión andina, utilice signos icónicos y rasgos pertinentes de una o varias culturas de Sudamérica, objeto de estudio de la asignatura. El artículo es una crónica del proceso de trabajo co-creativo, cuyo principal resultado es la fundamentación filosófica conceptual y descripción de la simbología de la marca Chakiñan. A manera de conclusión se abordan las ventajas del sistema de diseño colaborativo en oposición al clásico sistema competitivo.

Palabras clave: chakiñan; co-creación; diseño colaborativo; marca corporativa.

\section{ABSTRACT}

The emerging scientific journal of sciences and humanities, Chakinan, will require a visual identifier that represents its distinctiveness, acquired notoriety among the academic community and acquired a solid position in the context of indexed journals. To accomplish this task, the collaborative design system was proposed, with the contribution of eight students who studied the Andean Design course, in the period october 2016 - march 2017. In the development, Bruce Archer's project methodology was used, which consist of four phases: analytical, creative, formalization of the idea and implementation stage. Among the determining factors was the considered the need for a visual identifier to reflect the Andean worldview, using iconic signs and relevant features from one or several cultures of Mesoamerica and / or South America, aim of study. The article reflects the the co-creative work process, whose main result is the philosophical and conceptual foundation and description of the symbolism of the Chakinan brand. In conclusion, the advantages of the collaborative design system is in contrast to the common competitive system.

Keywords: chakiñan; co-creation; collaborative design; corporative brand.
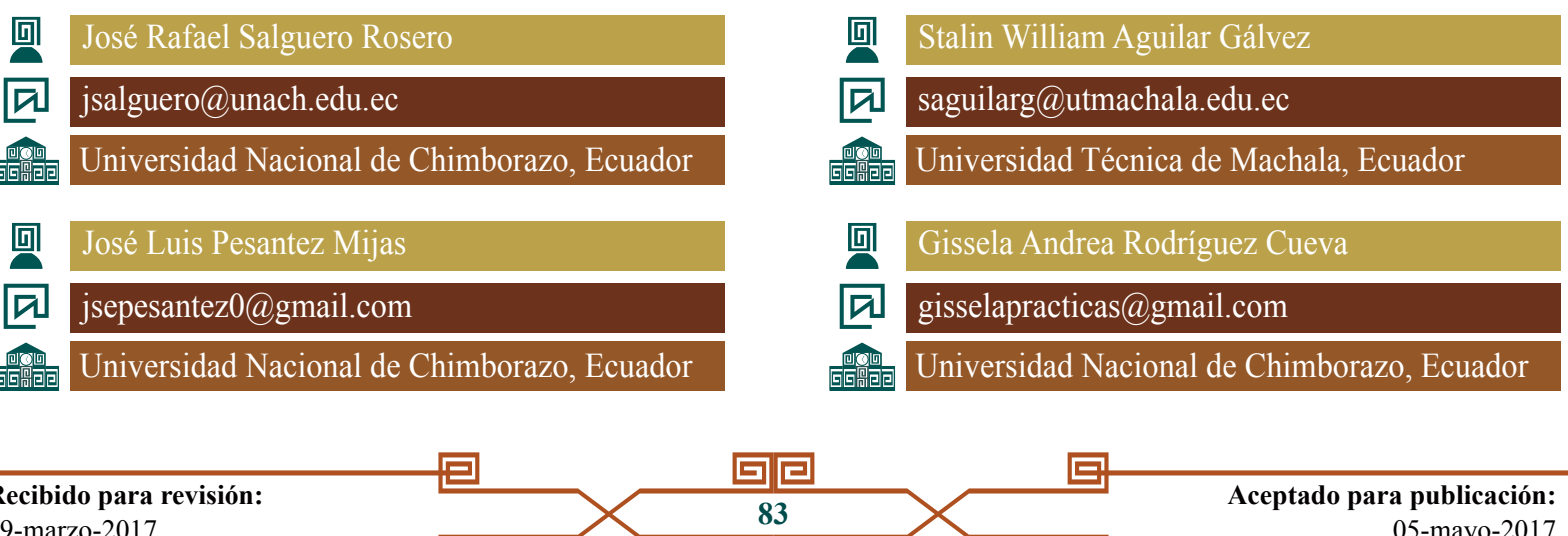


\section{INTRODUCCIÓN}

Crear una marca, no solo representa escoger una palabra eufónica, una tipografía legible, un ícono denotativo o connotativo y armonizar el color. La marca debe identificar, caracterizar y canalizar un mensaje visual a través del cual, las audiencias o públicos objetivos a los que se dirige, piensen en sus productos o servicios cuando interactúan con ella.

Keller (2008), explica que "la marca genera cierta cantidad de conciencia, reputación y prominencia en el mercado", entonces, cuando un equipo creativo o diseñador se enfrenta a un problema marcario, está consciente que diseñar una marca es un proceso que requiere una alta dosis de conocimiento, tanto como creatividad; además requiere esclarecer cuales son las características homogéneas del público objetivo con el que establecerá comunicación.

Para Chaves y Bellucia (2006:52-53), "la marca es un signo visual cuya función principal es identificar al emisor". Así, el equipo creativo debe insertarse en un proceso investigativo cuyo primer objetivo es establecer los criterios o elementos que constituyen la identidad de ese emisor. Estos criterios serán a futuro, los constructos multidimensionales que evidencien un posicionamiento, valiéndose de valores funcionales como emocionales de la organización, en coherencia con las necesidades de sus consumidores.

Diseñar una marca es un proceso interesante e importante, ya que pone en evidencia las destrezas del diseñador o equipo creativo. No solo las habilidades prácticas de boceteo, redibujo, manejo cromático y tipográfico, también habilidades cognitivas sobre temáticas tan diversas como las categorías y reglas compositivas, la construcción de perfiles de públicos objetivos o segmentos de mercado, las tendencias de arte y diseño, la psicología del color, entre otros.
Chaves (2004:12), define a la marca como "la representación más concentrada, espontánea, nocional y emocional de una organización, que ha adquirido notoriedad, reputación y personalidad". En base a lo citado, construir la marca va más allá de diseñar el identificador visual; esto solo constituye el punto de partida. Un constructo visual marcario esencialmente está compuesto por el nombre o fonotipo, la parte de la marca que se pronuncia y constituye su identidad verbal; el logotipo, comprendido como la representación gráfica del nombre, que forma parte de la identidad visual; y los grafismos, que son aquellos íconos, colores y más factores no pronunciables, que la complementan.

La complejidad es aún mayor, dado que, una vez construido el identificador visual, inicia un proceso definido por Chaves $\left(20^{\circ} 4: 24\right)$, como "el mito de la marca gráfica como transmisora de valores". El mismo autor explica que esta actividad debería ser la más lógica y natural, pero es la más difícil de lograr. Es en este punto donde converge lo nocional y emocional de la organización a la que representa la marca. El identificador visual irá ganando notoriedad entre sus públicos y ligándose a valores que pueden ser parte de su identidad como tal, pero también pueden ser adjudicadas por las percepciones del público con el que interactúa. Así, apoyándose en la sumatoria de trayectoria, historia, notas de prensa, trato, calidad, multiplicidad de servicios, avisos, entre otros; la marca gana un espacio en la memoria del público, construyendo su imagen. Esas interacciones y experiencias con la marca gráfica producen la equivalencia entre marca y posicionamiento.

Conociendo la necesidad de sustento filosófico u ontológico que requiere un identificador visual, capaz de posicionarse entre el exigente público de investigadores redacto- 
res y lectores de revistas indexadas, Carretero (2016:4), expresó en el primer editorial que, "Chakiñan pretende ser la puerta al diálogo que hace posible el equilibrio, el objetivo de la vida en el mundo andino. La publicación nació como alternativa de respuesta a la necesidad de establecer vínculos entre la diversidad, la pluralidad y la interdisciplinariedad del ser humano con su entorno". Si bien la publicación nació con una identidad verbal, con una alta carga simbólica, el desarrollo de la identidad visual y la estrategia de comunicación fue el trabajo encargado al equipo creativo.

Convencionalmente, la alternativa más usual para la generación de marcas es el clásico sistema competitivo, donde el, o los gestores, llaman a un concurso entre varios proponentes y entre las propuestas generadas se escoge la que cumpla con mayor número de parámetros técnicos. En cambio, para este caso se trabajó en un proceso co-creativo o de diseño colaborativo. Los participantes fueron ocho estudiantes de la asignatura de Diseño Andino, que cursaron el séptimo semestre de formación en Diseño Gráfico en la Universidad Nacional de Chimborazo. El profesor condujo el proceso en todas sus fases, hasta llegar a un primer producto, el manual básico de marca. Como se especificó en los párrafos anteriores, esta es solo una primera fase del proceso para posicionar a Chakiñan, como una marca reconocida entre el contexto de las revistas indexadas latinoamericanas, en el ámbito de las ciencias y humanidades.

Los objetivos del artículo son, socializar el proceso constructivo, sustentar la fundamentación filosófica conceptual y describir la simbología de la marca Chakiñan. Además, a manera de conclusiones se detallarán ventajas del sistema de diseño colaborativo en oposición al sistema competitivo.

\section{METODOLOGÍA}

Para el desarrollo de la marca Chakiñan se empleó el método sistémico de Archer (s/f) que consta de cuatro fases. La primera es la fase analítica, parte de la recopilación de datos, sigue con el ordenamiento de la información recolectada, evaluación de los datos sobresalientes, definiendo condicionantes de utilidad y concluye con la jerarquización de la información que aportará al proceso de diseño.

La segunda fase es la creativa, inicia tomando como base la información sintetizada en la fase anterior para desarrollar las ideas que aportarán a la solución. Archer recomienda en esta fase formular ideas rectoras, debatirlas entre el equipo creativo y tomar partido por una idea básica.

La tercera fase concierne a la formalización de la idea, donde hace falta una reflexión crítica sobre la idea básica que se acordó. Concluye con la verificación de la idea seleccionada, haciendo los ajustes necesarios para garantizar una propuesta efectiva con altos estándares técnicos y estéticos.

La cuarta y última fase es la ejecutiva, inicia haciendo una nueva valoración crítica de la idea seleccionada. De ser necesario se hacen ajustes a la idea, para iniciar el desarrollo. Así se llega al proceso interactivo entre el equipo creativo, que concluye en la materialización de una propuesta de diseño; para el caso, la propuesta de marca.

Complementa la metodología de diseño colaborativo, descrito por Sanders (2009:1-3), como la "unión de la creatividad de varios diseñadores que trabajan juntos en el proceso de elaboración de un diseño". Este procedimiento multiplica la creatividad a través del trabajo colectivo denominado 
co-creación, mediante el cual se obtiene un resultado de mayor calidad.

\section{RESULTADO Y DISCUSIÓN}

\section{Sustentación filosófica}

El constructo visual marcario se fundamenta en la Cosmología Andina que expone la distribución de todos los elementos de la naturaleza en pares duales representados por los astros, sol, inti, y luna, killa; los momentos de cambio, mañana y tarde, las constelaciones de siembra, Catachillay, y de cosecha, Choqechinchay, los seres en general, hombre, qhari y mujer, warmi, los sustentos naturales, tierra y agua, entre otras.

Otro principio filosófico andino, representado en el Altar de Coricancha, es la tripartición del Universo en tres planos integrados que se comunican, transforman y reciprocan entre sí; Hanan Pacha, donde está el ser superior, el ordenador del universo, los principales astros, los espíritus del cielo, el mundo de arriba, el mundo que está fuera del aquí y del ahora. Kai Pacha, el mundo del aquí y del ahora, de todo lo que existe en este plano, aquí coexisten los hombres y mujeres en perfecta armonía con la tierra, las plantas, los animales. Y el Uku Pacha, el mundo de lo interior, de lo profundo. Aunque es el mundo de los no nacidos, también es el mundo donde todo nace.

La Cosmología Andina se perfecciona con el principio de la complementariedad y la relacionalidad entre lo de arriba y abajo, el Hanan Pacha y el Urin; entre la izquierda y la derecha, la Luna y el Sol, la nube y el rayo, la lluvia y el arco iris, las plantas y el suelo, la estrella vespertina y la estrella matutina (Venus en ambos casos). En el centro está la Chakana, que constituye el puente cósmico de transición entre los cuatro cuadrantes, arriba abajo, derecha e izquierda. Es también el vínculo para que algo funcione, ya que todo necesita de su par o complemento.

Otro principio filosófico andino considerado es la ciclicidad del tiempo. Estermann (1998:88) explica que "el tiempo andino no es unidireccional, de pasado a futuro, sino bi- o multidireccional; para la racionalidad cíclica el futuro realmente está atrás y el pasado adelante, pero también viceversa". Citados principios filosóficos, o en palabras de Estermann, "ecosofía o pachasofía" constituyen la información que sustentará la propuesta gráfica.

\section{Ideas rectoras que sustentan la marca}

\subsection{Fonotipo}

Chakiñan, traducido literalmente del quechua significa sendero. Pero desde la cosmovisión andina, chakiñan es una red que interconecta los pueblos, es decir, un punto de encuentro entre diversidad de culturas, de conocimientos. Es un sendero en el cual el ser humano se encuentra con otro, e interconecta su forma de ver e interpretar el mundo generando conocimiento.

Implícitamente la identidad verbal de Chakiñan invita a interconectar a la sociedad en base al desarrollo de la ciencia, visibilizar las reflexiones expresadas en las investigaciones de los autores en los cuatro puntos cardinales. Cumple con la visión de su equipo editorial que pretende que la revista sea "la puerta al diálogo de la vida en el mundo andino, la alternativa de respuesta a la necesidad de establecer vínculos entre la diversidad". 


\subsection{Concepción semiótica de los íconos que conforman el grafismo}

\subsubsection{Principio de dualidad y oposición} andina: la cosmovisión andina específica la complementariedad existente entre los cuatro cuadrantes, izquierda derecha, arriba abajo; así como los elementos reflejados según su visión cosmológica en la imagen del Altar o Estela de Coricancha. El pensamiento andino expone una paridad complementaria conocida como yanan-tinkuy. Esta paridad coincide en la cultura mesoamericana Maya-Quiche, así como en la cultura sudamericana Puquina-Inka.

2.2.2. Hunab Ku: el término proviene de tres voces mayas, Hun que se traduce como Uno, $A b$ que significa existir y $K u$ que se traduce como Dios. Para la cultura Maya, Hunab Ku constituye el Absoluto, la deidad única, el Gran Padre y la Gran Madre. Posee tres aspectos: El Inmanifestado, el Estado de Vida que anima todo ser y la materia caótica, atómica y seminal; consideran que el universo surgió de sus entrañas.
2.2.3. Willodmawe Nimin: es un ícono que representa a la serpiente. Podkul (s/f), antropólogo que estudió los textiles de la cultura Mapuche, lo define como un diseño que se enrolla sobre sí mismo, representando un abrazo. Se podría también relacionar con la concepción cíclica del tiempo, desde la cosmovisión andina. En las culturas ecuatorianas también aparece la serpiente como animal procreador, específicamente en la cultura Cañari, quienes se denominan sus hijos.

2.2.4. Espiral Andino: se asocia a los ciclos de la vida. La cultura Mapuche en Chile lo define como "el avance hacia el pasado y el regreso al futuro" (Gavilán 2012:55). Refiere a lo que Estermann denomina "tiempo andino" que no es unidireccional sino bi- o multidireccional.

\subsection{Primeras propuestas conceptuales} A continuación, las siguientes figuras muestran las propuestas conceptuales.

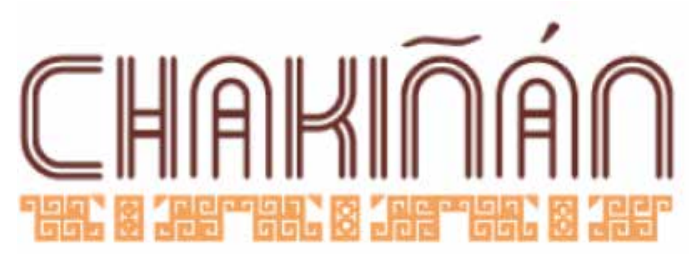

Figura 1: Propuesta conceptual basada en la Cultura Cañari, fase Tacalshapa Fuente: elaboración propia.

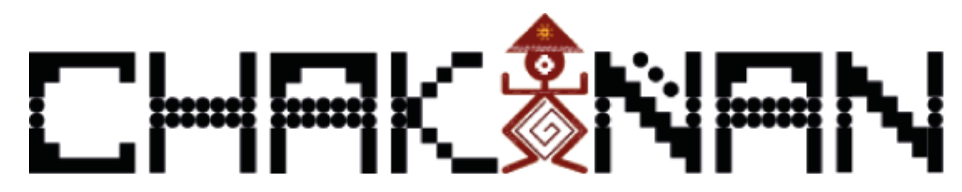

Figura 2: Propuesta conceptual basada en la Cultura Cañari, fase Narrío Fuente: elaboración propia. 


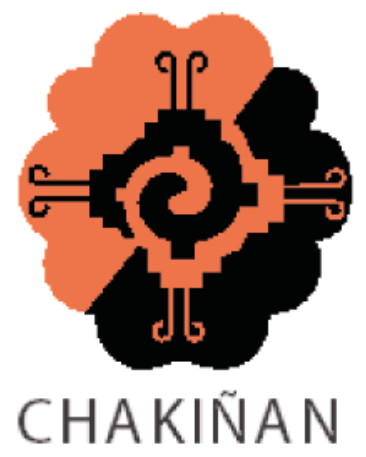

Figura 3: Propuesta conceptual basada en la cultura Maya - Quiche Fuente: elaboración propia.

Cumpliendo los pasos determinados en la metodología de Archer (s/f), se procedió a seleccionar la idea mejor conceptualizada. En consenso se aprobó la expuesta en la figura 3; pero se consideró tomar en cuenta elementos icónicos conceptuales de las dos propuestas descartadas. Puntualmente, el Nawpa espiral y delimitador del Pacha (Tiempo/Espacio); el Ojo Andino u Ojo del Conocimiento, conocido como Ollín $y$ Willodmawe Nimin, que se enrolla sobre sí misma (abrazo/protección).

El principal criterio semiótico considerado para la elección, es el principio de la dualidad expresado por el ícono, base de la concepción filosófica andina; también la riqueza conceptual del Hunab $\mathrm{Ku}$ como el elemento iniciador de todo lo existente. Además, implícitamente el ícono refleja la cruz andina o chakana, que puede comprenderse como Chaka $=$ cruce - Hanaq $=10$ Superior, es decir cruce a lo Superior; desde un sentido académico, el conocimiento contribuye a comprender el universo y su orden.

\subsection{Materialización de la propuesta de marca}

\subsubsection{Estructura compositiva}

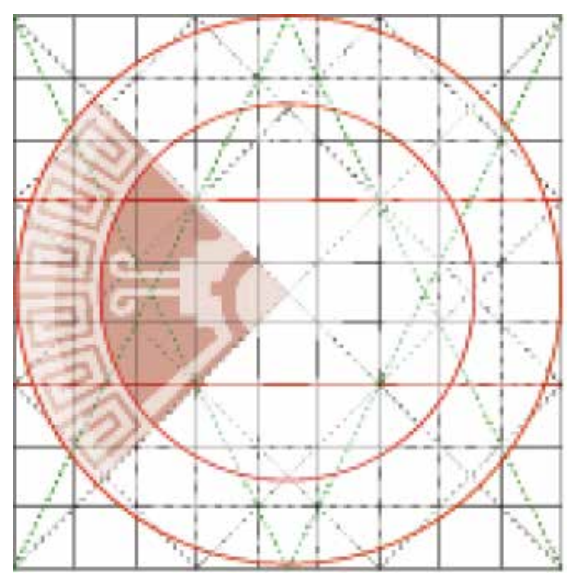

Figura 4: Estructura compositiva Fuente: elaboración propia.

La figura 4 evidencia la estructura compositiva utilizada para el ordenamiento de grafismos que constituyen la marca. Se aplica la ley de tripartición armónica terciaria, definida por (Milla 1990:95-96) como "el resultado del juego de diagonales del cuadrado con las diagonales del rectángulo $1 / 2$, cuyas cruces permiten ubicar los puntos del trazo de las ortogonales respectivas". Desde una visión semántica se comprendería la visión andina de dividir el espacio en tres partes que representarían el Hanan Pacha, Kai Pacha y Uku Pacha. Además el punto medio permite generar un equilibrio simétrico que representa la complementariedad de la dualidad. 


\subsubsection{Principios compositivos de Diseño}

En la propuesta de la figura 5, se aplican los citados principios: Equilibrio Radial, comprendido como aquel que da lugar a un movimiento giratorio o de rotación, que toma como centro un punto, sobre el cual

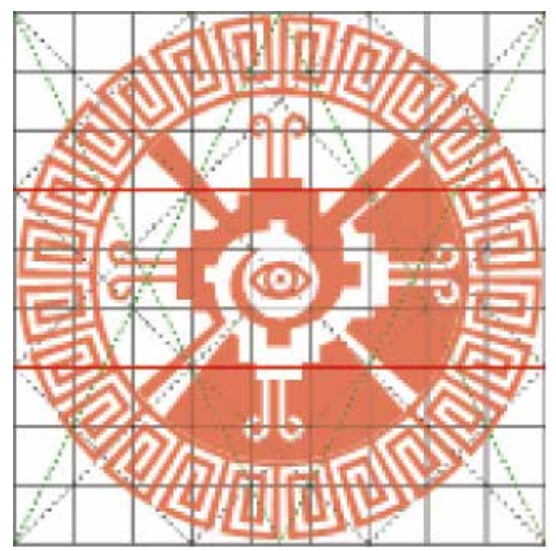

Figura 5: Principios compositivos de Diseño Fuente: elaboración propia. rotan los elementos, logrando así la compensación de fuerzas. El eje es la pupila del Ojo del Conocimiento u Ollín. Repetición, manifestado en los círculos concéntricos que agrupan los elementos del grafismo, así como el ícono de Willodmawe Nimin, que representa lo cíclico del tiempo. Ritmo, representado por una ampliación progresiva y regular de un elemento, partiendo del Ojo como eje central, lo que da un sentido dinámico; obliga al espectador a recorrer la secuencia presentada. Dirección, consiste en enlazar entre los tres mundos, el marco que contiene las formas cercanas.

\subsubsection{Propuesta grafismo final}

En la figura 6 se puede apreciar la propuesta de grafismo final.

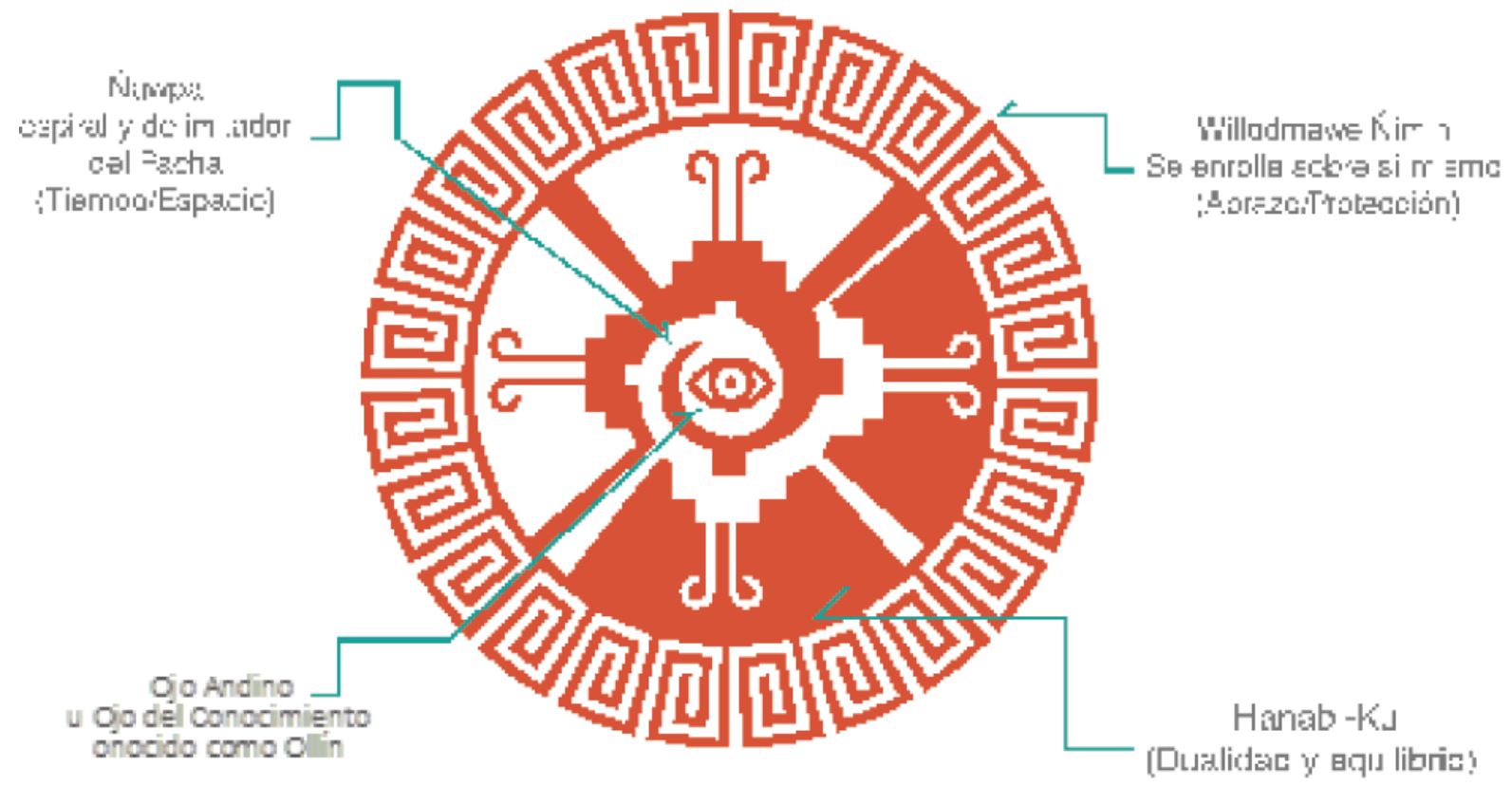

Figura 6: Propuesta grafismo final

Fuente: elaboración propia. 


\subsubsection{Variantes cromáticas y tipografía}

Para la correcta aplicación del identificador visual se determinaron como colores corporativos, los siguientes: Pantone $8863 \mathrm{C}$ y Pantone 3272C (como se aprecia en la figura 7). La tipografía corporativa es la

Proxi 9 y la tipografía complementaria la Helvética Light. La opción cromática dependerá de los fondos utilizables, el manual de marca determina los colores complementarios y las variantes de color del identificador visual.

\subsubsection{Evaluación de cumplimiento de los parámetros de calidad de marca}
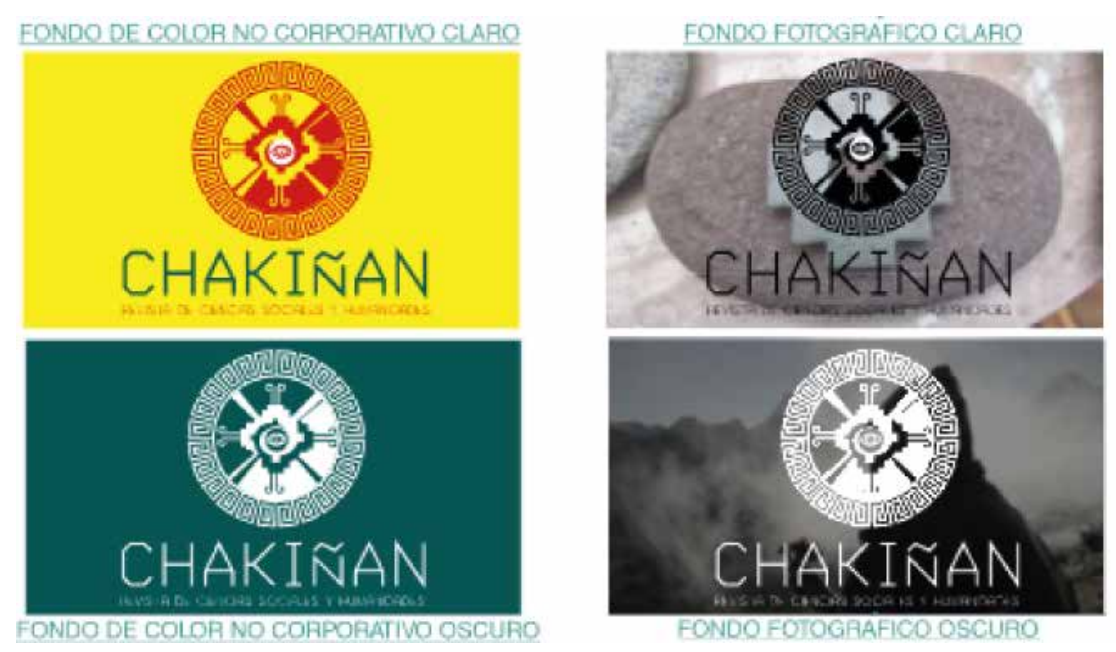

Figura 7: Variantes cromáticas y tipografía Fuente: elaboración propia.

Tabla 1: Ficha de evaluación de parámetros de calidad de marca

\begin{tabular}{|c|c|c|}
\hline Parámetro técnico & CHAKINAN & Observaciones \\
\hline Individualidad & & $\begin{array}{l}\text { Los grafismos utilizados son íconos simbólicos } \\
\text { de las culturas mesoamericanas y } \\
\text { sudamericanas; sin embargo constituye un } \\
\text { constructo visual original. }\end{array}$ \\
\hline $\begin{array}{l}\text { Pertinencia tipológica } \\
\text { y estilística }\end{array}$ & & $\begin{array}{l}\text { El grafismo se ajusta al perfil de la organización } \\
\text { que la regenta. Posee una sustentación filosófica } \\
\text { que refleja la identidad de la revista para la cual } \\
\text { fue creado; además se complementa con el } \\
\text { fonotipo y el logotipo. }\end{array}$ \\
\hline Legibilidad & & $\begin{array}{l}\text { Si bien el fonotipo es eufónico y la tipografía } \\
\text { escogida para el nombre es legible, el grafismo } \\
\text { tiene una complejidad alta, lo que exige al } \\
\text { usuario mayor tiempo de observación y } \\
\text { reflexión para comprender la marca. Podría } \\
\text { dificultarse la pregnancia. }\end{array}$ \\
\hline
\end{tabular}




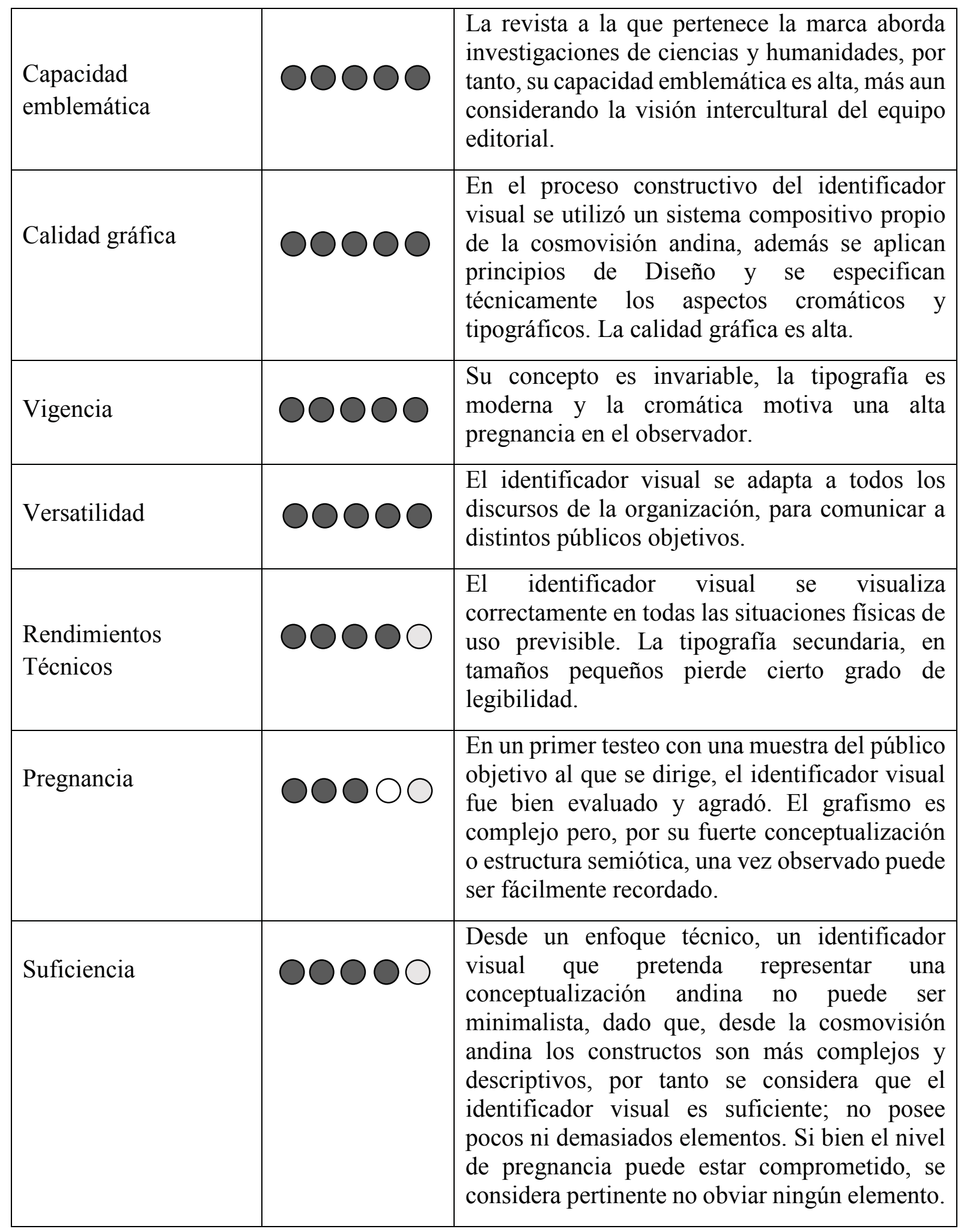

Fuente: elaboración propia.

La tabla 1 muestra una síntesis de autoevaluación bajo los parámetros técnicos propuestos por Norberto Chaves y Raúl Bellucia (2006). Como se evidencia, el iden- tificador visual propuesto cumple parámetros de calidad que garantizarán el posicionamiento a mediano plazo.

El proceso co-creativo exige llegar a consen- 
sos en todas las fases, partiendo desde la determinación del fonotipo, aunque para el caso, el nombre ya estuvo formulado por los gestores. En el desarrollo icónico primó la sustentación de cada proponente y la carga semiótica, cada participante expresó sus observaciones que contribuyeron en el perfeccionamiento del grafismo. Para la fase de aplicación cromática cada participante presentó dos opciones de aplicación, entre gamas, armonías, contrastes y monocromías. A través de la observación de la marca gráfica impresa en tres tamaños, se valoró bajo los parámetros técnicos expresados en la tabla 1. Cada participante valoró en una escala numérica

de uno a cinco. Una vez contabilizados, los valores se expresaron cromáticamente, añadiendo las conclusiones por cada ítem valorado. A la par, se cumplió un proceso de valoración icónica y cromática, con los integrantes del comité editorial y científico de la revista. La marca gráfica mejor valorada es la que adoptaron los gestores.

Como se evidencia en el proceso descrito, crear una marca encierra un proceso de alta complejidad. La marca Chakiñan busca ser identificada y reconocida entre la comunidad académica, no solo de la universidad a la que pertenece, sino en el contexto latinoamericano y mundial, a través de las redes de divulgación científica, en las diversas plataformas indexadas. Se espera que sea valorada positivamente, no solo desde el punto de vista gráfico o visual, sino que, los diversos soportes gráficos, físicos y digitales, así como el contenido, provoquen una adhesión de los públicos a los que se dirige; entre ellos, docentes y estudiantes investigadores, árbitros, redactores y lectores.

Chakiñan busca diferenciarse de su competencia desde su proceso de concepción. $\mathrm{Su}$ oferta de valor se fundamenta en abrir un sendero para fomentar la investigación y reflexión relacionadas con las ciencias sociales y las humanidades, donde se aborden estudios del ser humano como ente social. Una particularidad considerada desde la generación del fonotipo es la valoración de la lengua materna, quechua. Para complementar el fonotipo escogido, el grafismo armoniza la identidad verbal, dado que es un ícono altamente pertinente, que se sustenta en la pachasofía, evidenciando signos acuñados por la cultura Maya - Quiche, en concordancia con signos de la cultura sudamericana Cañari y otras. Además, la política editorial consolida lo propuesto, con la publicación de al menos un artículo por edición en citada lengua

El identificador visual diseñado es el punto de partida para la consolidación de la imagen de Chakiñan. Es necesario acuñar la percepción pública sobre la revista, afianzándose en las audiencias, a través de comunicación corporativa de calidad, artículos publicados con alto rigor científico, apoyo a eventos académicos locales, nacionales e internacionales; de tal manera que progresivamente Chakiñan logre una influencia concreta que genere un posicionamiento en el competitivo mundo de las publicaciones indexadas.

A través del identificador visual diseñado se precisó los atributos de identidad de Chakiñan, el siguiente paso es afianzar el perfil estratégico de la publicación, y en base a este perfil, desarrollar un programa de gestión que conjugue y converja el trabajo de la dirección, el equipo editorial, el comité científico, los diseñadores, los gestores de marca, el equipo creativo de gestión publicitaria, las comunicaciones, las relaciones públicas y de prensa. Un trabajo armónico garantizará un posicionamiento positivo en un periodo a corto, mediano y largo plazo. 
Como lo expresa Huerta (2014:3), "la irrupción del diseño colaborativo como paradigma está cambiado el panorama de la práctica del diseño". La co-creación evidencia el dominio de "creatividad colectiva", que fortalece la interacción entre profesionales, nutre el trabajo investigativo, aflora propuestas con altos estándares de calidad que mejoran sesión tras sesión.

Sanders (2009:2-3), explica que el diseño colaborativo "requiere de nuevas herramientas, métodos y un nuevo lenguaje del diseño". El diseñador, acostumbrado a trabajar individualmente, rompe su paradigma y adopta nuevas actitudes para trabajar en equipo, lo que permite autoevaluarse y evaluar a sus pares; en este proceso todo el equipo adquiere nuevos aprendizajes, mejorando notablemente la calidad de sus propuestas.

Para que el diseño colaborativo o co-creación funcione, es necesario que el equipo tenga claro el objetivo en común; para el caso de estudio, el diseño de la marca Chakiñan. El diseñador incluye nuevos "socios creativos" en un contexto en el cual estaba acostumbrado a trabajar individualmente, si bien se pierde el control total sobre las decisiones a ejecutarse, se abre un espectro amplio de creatividad sumando a la de su equipo. El resultado logrado evidencia la funcionalidad del proceso.

\section{CONCLUSIONES}

El identificador visual de la marca Chakiñan cumple satisfactoriamente con 10 parámetros técnicos; un consistente punto de partida para la consolidación de la imagen y el posicionamiento entre sus públicos objetivos. Refleja la identidad de la revista a la que identifica y los valores de los gestores de marca. Utiliza elementos singulares tanto en el fonotipo, el logotipo como en el grafismo, lo que garantizará su reconocimiento y pregnancia.

Los diseñadores en formación consolidaron destrezas para el trabajo colaborativo, aprendieron a desarrollar procesos de creatividad colectiva, mejoraron sus destrezas metodológicas proyectuales y asimilaron la importancia de la auto y co-evaluación. 


\section{Unach}

\section{REFERENCIAS \\ BIBLIOGRÁFICAS}

Carretero, P. (2016). Editorial. Revista Chakiñan, (1), pp. 1. Recuperado de http://chakinan.unach.edu.ec/index.php/chakinan/article/view/5/2

Chaves, N. (2004). La imagen Corporativa. México D.F., México: Ediciones G. Gill.

Chaves, N. y Bellucia, R. (2006). La Marca Corporativa. Buenos Aires, Argentina: Paidós.

Estermann, J. (1998). Filosofia Andina. Estudio intercultural de la sabiduría autóctona andina. Quito, Ecuador: Ediciones Abya Yala.

Gavilán, M. (2012). El pensamiento en espiral. El paradigma de los pueblos indígenas. Nuke Mapuförlaget Working Paper. (40), 55-56.

Huerta, E. (2014). La Co-Creación y el Diseño Colaborativo. Recuperado de http://www.esdi.es/content/pdf/articuloweb_esdi-4_ehuerta180913.pdf

Milla, Z. (1990). Introducción a la semiótica del diseño precolombino andino. Lima, Perú: Editorial Asociación de investigación y comunicación cultural Amaru Wayra.

Keller, K. (2008). Branding: administración estratégica de marca. México DF, México: Prentice Hall.

Sanders, E. (2009). A Social Vision for Value Co-creation in Design, Open Source Business Resource, Value Co-Creation. Recuperado de http://timreview.ca/no$\mathrm{de} / 310$

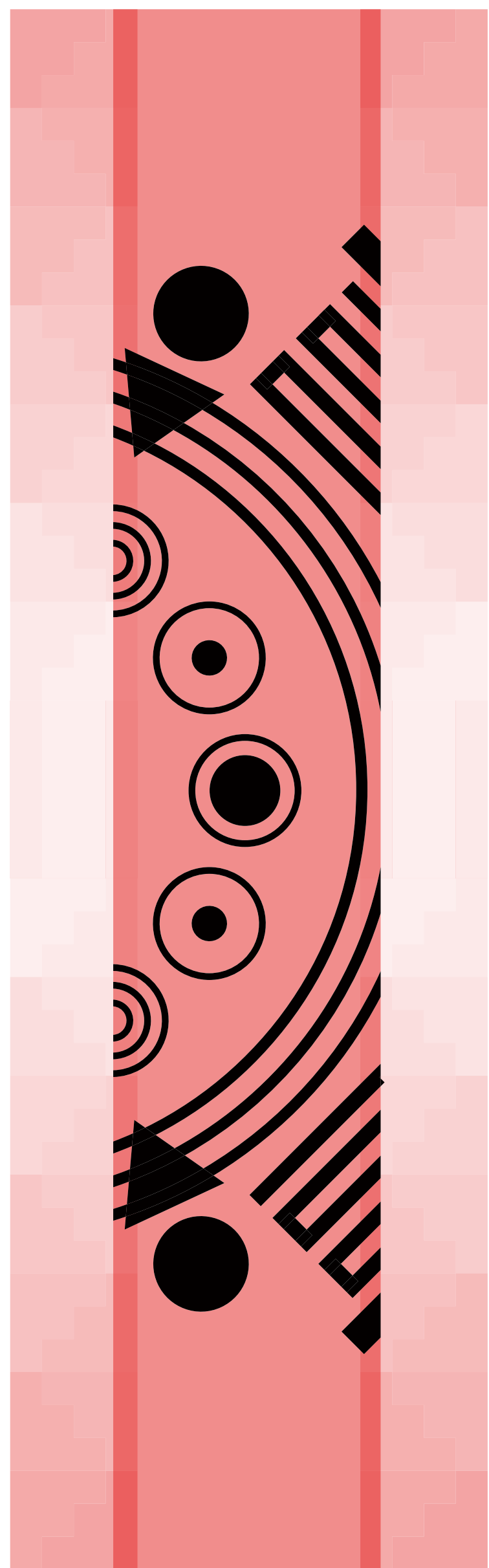

